\title{
Enabling Healthcare in Smart Homes: The SPHERE loT Network Infrastructure
}

Elsts, Atis; Fafoutis, Xenofon; Woznowski, Przemyslaw; Tonkin, Emma; Oikonomou, George; Piechocki, Robert; Craddock, lan

Published in:

IEEE Communications Magazine

Link to article, DOI:

10.1109/MCOM.2017.1700791

Publication date:

2018

Document Version

Peer reviewed version

Link back to DTU Orbit

Citation (APA):

Elsts, A., Fafoutis, X., Woznowski, P., Tonkin, E., Oikonomou, G., Piechocki, R., \& Craddock, I. (2018). Enabling Healthcare in Smart Homes: The SPHERE loT Network Infrastructure. IEEE Communications Magazine, 56(12), 164-170. https://doi.org/10.1109/MCOM.2017.1700791

\section{General rights}

Copyright and moral rights for the publications made accessible in the public portal are retained by the authors and/or other copyright owners and it is a condition of accessing publications that users recognise and abide by the legal requirements associated with these rights.

- Users may download and print one copy of any publication from the public portal for the purpose of private study or research.

- You may not further distribute the material or use it for any profit-making activity or commercial gain

- You may freely distribute the URL identifying the publication in the public portal 
Elsts, A., Fafoutis, X., Woznowski, P. R., Tonkin, E. L., Oikonomou, G., Piechocki, R., \& Craddock, I. (2018). Enabling Healthcare in Smart Homes: The SPHERE IoT Network Infrastructure. IEEE Communications Magazine, 56(12), 164-170. https://doi.org/10.1109/MCOM.2017.1700791

Peer reviewed version

Link to published version (if available):

10.1109/MCOM.2017.1700791

Link to publication record in Explore Bristol Research

PDF-document

This is the author accepted manuscript (AAM). The final published version (version of record) will be available online via IEEE. Please refer to any applicable terms of use of the publisher.

\section{University of Bristol - Explore Bristol Research}

\section{General rights}

This document is made available in accordance with publisher policies. Please cite only the published version using the reference above. Full terms of use are available:

http://www.bristol.ac.uk/pure/about/ebr-terms 


\title{
Enabling Healthcare in Smart Homes: The SPHERE IoT Network Infrastructure
}

\author{
Atis Elsts $^{\dagger}$, Xenofon Fafoutis ${ }^{\dagger}$, Przemyslaw Woznowski, Emma Tonkin, \\ George Oikonomou, Robert Piechocki, and Ian Craddock
}

\begin{abstract}
Healthcare professionals currently lack the means to gather unbiased and quantitative multi-modal data about the long-term behaviors of patients in their home environments. SPHERE is a multi-modal platform of non-medical sensors for behavior monitoring in residential environments that aims to overcome this major limitation of healthcare provision through using the inherently cost-efficient and scalable technologies of the Internet of Things (IoT). One of SPHERE's key tasks is to help to bring the next-generation low-power wireless networking and sensing technologies from the lab to the field by applying them in real-world environments. In this article we describe the highlights of SPHERE's system requirements, architecture, practical challenges, as well as of the design and deployment lessons learned. By leveraging novel IoT technologies such as the IEEE 802.15.4 TSCH network protocol, SPHERE has achieved successful initial deployments in twelve volunteer houses at the time of writing.
\end{abstract}

\section{INTRODUCTION}

Health systems are under severe pressure due to the continuously aging population and the related increase of chronic illnesses. In this landscape, early-detection of medical conditions and cost-effective, personalized treatment are paramount for the long-term sustainability of health care provision and for making efficient use of the health system's limited resources. There is increasing evidence that behavior plays a critical role in the development of chronic health conditions [1]. For example, sleep disturbances have been linked with an increased risk of depression [2], while a measurable decline in the more complex activities of daily living, such as cooking, is linked with the onset of dementia [3]. Yet, healthcare professionals lack an unbiased and quantitative mechanism to assess the long-term behaviors of their patients.

The Internet of Things (IoT) is posed to become a key enabling technology with respect to residential healthcare applications [4], offering more scalable, cost-effective, and unobtrusive health-relative sensing compared to hospital visits and more objective measurements compared to patient selfreporting. SPHERE (a Sensing Platform for HEalthcare in a Residential Environment) is a multipurpose, multi-modal platform of non-medical home sensors that aims to collect data that would enable researchers to learn the behavior and routines of the residents, to identify suspicious abnormalities, to facilitate timely interventions, and to conduct data-rich clinical studies [5]. SPHERE is composed of three core sensing technologies: wearable sensor nodes (one per resident); environmental sensor nodes that capture the home's environmental

The authors are with the University of Bristol. Xenofon Fafoutis is also with the Technical University of Denmark. ${ }^{\dagger}$ The first two authors contributed equally to this work. parameters (one per room); and video sensors for activity recognition with video analysis. The first two form the IoT subsystem of SPHERE, which is the focus of this article. The IoT subsystem utilizes recent advances in hardware, which have resulted in energy-efficient, low-cost sensors and microcontrollers, and advances in protocols and software which have made low-power wireless communications interoperable, reliable, predictable, and robust to interference and signal fading [6].

Unlike many smart home health systems evaluated in controlled environments [7], [8], SPHERE is intended to operate unsupervised in the wild. At the time of writing, the SPHERE platform has been deployed in twelve homes of volunteer participants in the Bristol area for up to 12 months; further deployments occur at an approximate rate of two homes per week, aiming for up to 100 homes in total. In this way, SPHERE contributes to the state-of-the-art by evaluating the suitability of health-related IoT technologies and system development practices for large-scale smart home deployments. This is a critical step to bring the next-generation smart home technologies from the lab to the field.

At a higher level, the central research question of SPHERE is whether long-term behavioral monitoring in our home environment using low-cost multi-modal sensors can enable more effective and cost-efficient health care provision. To this end, the research question of this article is whether the stateof-the-art IoT technology is able to provide a dependable data collection infrastructure that is suitable for long-term largescale deployments in the wild, and how it can be improved when not suitable. This article aims to highlight the most important lessons learned while designing and deploying the SPHERE platform and share them with the research community. To this end, this article makes the following contributions:

- We formulate IoT requirements in the context of SPHERE.

- We outline the IoT network architecture, hardware, and software used in SPHERE.

- We evaluate the suitability-for-purpose of several stateof-the-art IoT software technologies, for example, TSCH (Time Slotted Channel Hopping), which were previously not used in smart home projects of SPHERE's scale, and discuss the challenges encountered and enhancements added by us in order to satisfy the requirements.

Following up on earlier work describing the vision and ambitions of SPHERE [9], this paper reports on a complete, deployment-ready system. 


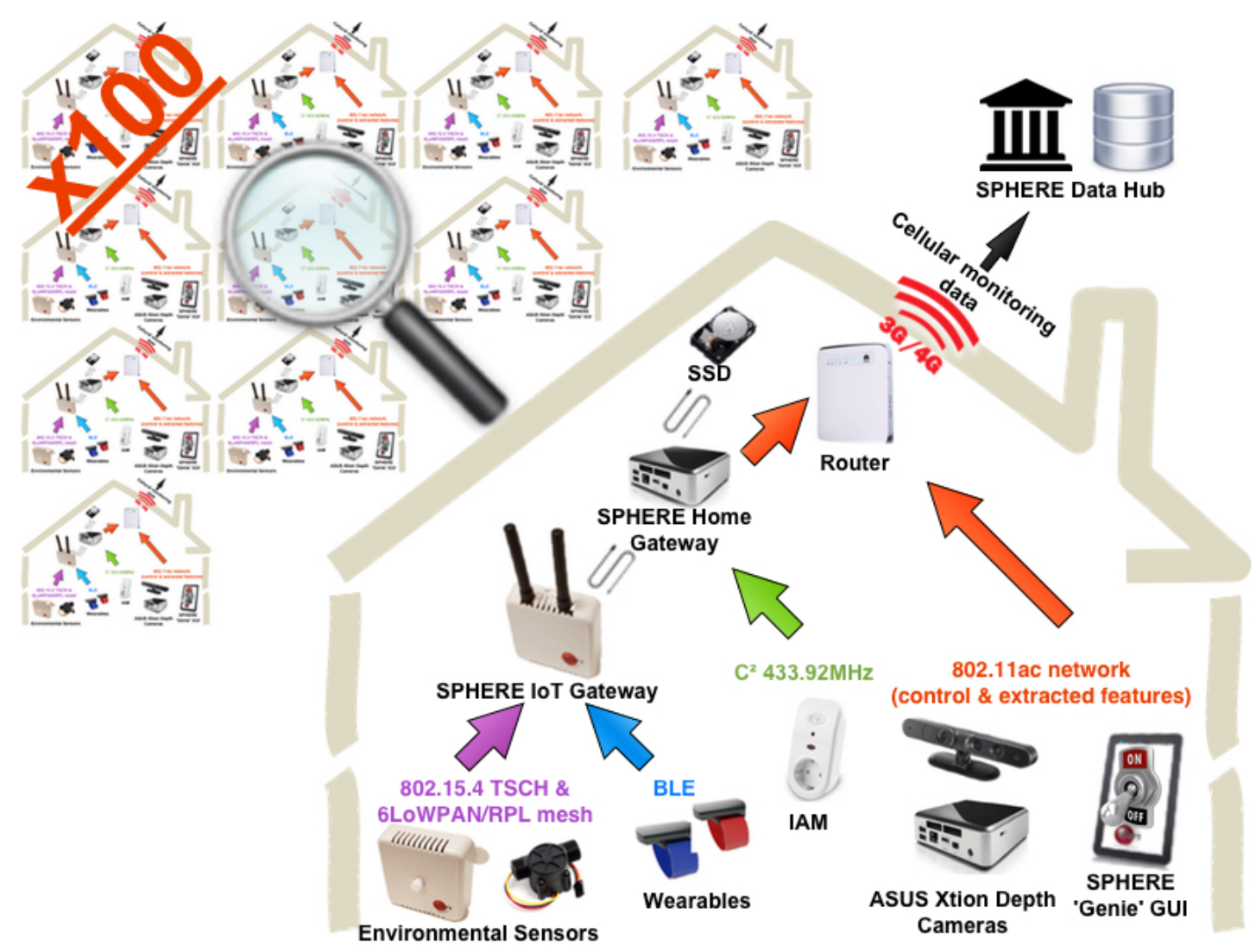

Figure 1: The SPHERE architecture supports the collection of data from multiple smart houses.

\section{THE SPHERE SYSTEM}

Existing smart home health systems make use of state-ofthe-art sensing technology, such as wireless sensor networks, wearable computing, and video cameras. However, they tend to use these technologies in isolation, aiming at addressing specific applications. In contrast, SPHERE attempts to be a general-purpose data collection platform that combines various sensing modalities. The data will be used as input for machine learning applications, such as daily activity recognition and room-level localization.

The SPHERE platform (Fig. 1) is heterogeneous: it combines low-power wearable sensors, environmental sensors, and video sensors; the latter are intended to support video-based activity recognition in the home. The SPHERE architecture combines these three sensing modalities with an in-home data aggregation layer.

The raw data from the environmental and wearable sensors are collected at a central server in each house, namely the SPHERE Home Gateway, via the SPHERE IoT Gateway which operates as the sink of the wireless sensor network. Health-related data is never transmitted over the Internet. It is securely stored in the home and physically collected by a technician at the end of the deployment.

\section{A. Requirements}

Unlike many smart home health systems [8], the SPHERE platform is not designed to run solely in controlled environments. Rather, it is intended to operate unsupervised in the wild. Installations are made in real homes, which in practice are hard-to-access private environments. This, along with the facts that SPHERE is public-facing and needs to scale to 100 deployments, leads to a set of requirements that is more common to an industrial application rather than to a typical research project.

The following requirements were engineered in a process that included all SPHERE's stakeholders, among which were machine learning researchers and the potential users of the technology. The general public was involved through extensive outreach activities, as well as through consultations with the SPHERE public advisory board. Last but not least, the system underwent several months of alpha-testing in the SPHERE house - a property rented by the project for this reason -, and beta-testing in two pilot deployments.

1) System-wide Requirements: The SPHERE IoT subsystem must satisfy the following requirements:

- Core functionality. The SPHERE system has to collect data from heterogeneous, spatially distributed sensor nodes that are connected wirelessly. The data has to be timestamped with an accuracy of less than $10 \mathrm{~ms}$ and stored on the SPHERE Home Gateway.

- Dependability, in terms of robustness, reliability, and predictability. The system ought to be robust and able to self-repair (i.e., provide automated recovery from faults); when that is not possible, it should fail gracefully in order to operate with limited maintenance, as visiting people's homes to repair the system is very undesirable. The system should not lose data; it should have near 


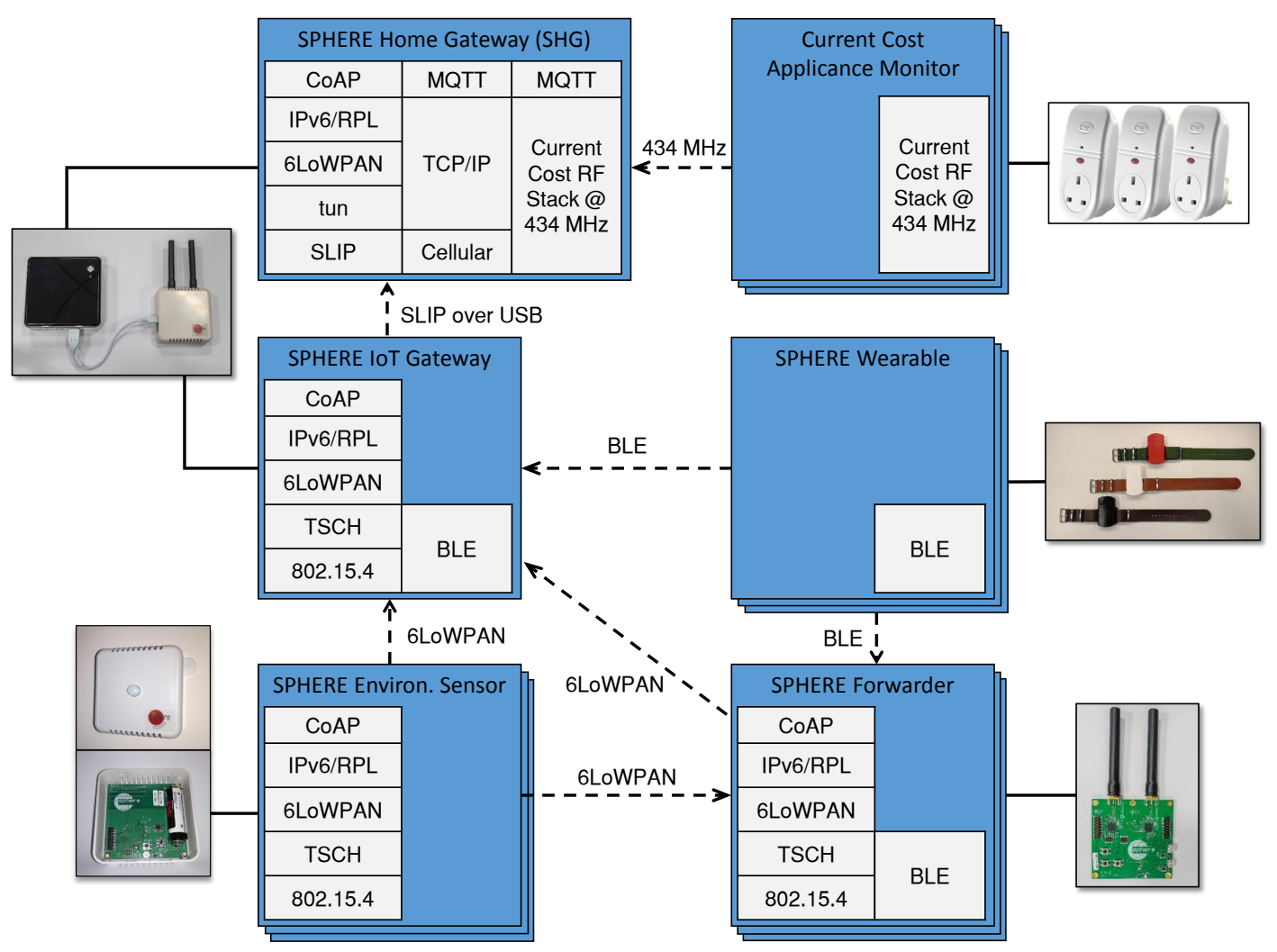

Figure 2: The SPHERE low-power IoT subsystem architecture interconnects environmental sensors, wearable sensors, and appliance monitors, using low power wireless technology.

100 percent packet delivery rate (PDR). The main challenges with regard to this during the development of the SPHERE system were low-level software and hardware problems [10]: stack overflows (solved by adding a stack usage checker) and failures to wake up from deep sleep modes (solved by adding an external watchdog to reboot such a node).

- Ethics compliance. At a more technical level this implies security and privacy. The health-related data in the project is deemed confidential; it is encrypted when stored as well as while in transit, must support anonymization, and must not include data from sensors that are too intrusive, for example, microphones. During the project we realized that the houses potentially could be de-anonymized based on seemingly innocuous data, for example, the barometric pressure sensor would effectively give out their location through altitude above sea level. To circumvent this, we now consider adding random offsets before sharing the anonymized SPHERE dataset.

- Ease of installation, maintenance, and use. A single system should be installable by non-expert personnel, in three hours or less. The system must provide run-time performance statistics, and should only require minimal effort from the participants to use. The nodes must be able to function as data forwarders, as single-hop wireless communication may not be sufficient. In SPHERE's outreach activities we learned that user acceptance is a critical issue, especially for the wearable enclosures which should be aesthetically pleasing and convenient to wear [11].

- Scalability and adaptability. The individual homes in the 100-home deployment each differ in size (number of rooms, number of participants, etc.), layout, and type of building materials used, all of which affects the performance of wireless protocols. SPHERE's infrastructure aims to adapt and self-repair during runtime; however, to optimize performance, SPHERE's firmware includes the number of devices per house as a configuration parameter. Hence, different houses use different firmware configurations depending on their sizes.

- Low cost. The devices use common-off-the-shelf components and avoid expensive sensors. For example, we took the decision to avoid deploying high-accuracy air quality sensors, despite significant interest from the potential participants, because they cost hundreds of pounds each.

- Interoperability. Standard low-power IoT protocols should be used whenever possible, in order to facilitate integration with third-party components in the future, and to simplify learning and sharing of the SPHERE system with new collaborators.

\section{2) Subsystem-specific Requirements:}

- Mobility and localization for the wearable nodes, from which continuous data delivery is expected as long as they are within the house. The communication stack must be able to deal with body and environmental shadowing, as well as localize the transmitter position in the house 
based on its signal strength. For indoor localization, it is important that multiple forwarder nodes pick up the signal, not just one.

- Energy efficiency for the environmental nodes, to completely avoid their recharging, and for the wearable nodes, to reduce their recharging frequency. We employ a number of low-level techniques [10] to optimize their lifetime.

\section{B. Sensing Platforms and Data Flows}

The SPHERE IoT subsystem is composed of a series of bespoke IoT devices with various sensing modalities (Fig. 2). All platforms are based on the CC2650 System-on-Chip, which supports two very common low power wireless technologies, namely, Bluetooth Low Energy (BLE) and 6LoWPAN (IPv6 over Low-Power Wireless Personal Area Networks).

A SPHERE Environmental Sensor is deployed in each room, capturing its environmental properties. This sensing platform is equipped with a series of sensors that measure temperature, humidity, air pressure and light levels. The SPHERE Environmental Sensor is also equipped with a Passive Infrared (PIR) sensor that detects mobility within the room. With a field of view of $82^{\circ}$ vertically and $94^{\circ}$ horizontally, a single Environmental Sensor is able to capture mobility within a large proportion of each individual room; yet, priority is given to doors in order to capture transitions between rooms. In addition, the SPHERE Environmental Sensor supports external sensors: a custom acoustic-based water flow sensor is attached to the Environmental Sensor that is deployed in the kitchen under the sink. The SPHERE Environmental Sensor operates on a $3.6 \mathrm{~V}$ AA battery and communicates to the SPHERE house over a 6LoWPAN network.

The wrist-worn SPHERE Wearable Sensor constitutes the second sensing modality. It is an acceleration-based activity sensor that is provided to each house resident, and uniquely identifies the individual within the house. It is also equipped with a gyroscope which can be activated and deactivated in software to ensure energy efficiency. The SPHERE Wearable is powered from a $100 \mathrm{mAh}$ Lithium-Polymer battery, which can be recharged wirelessly, using short-range inductive charging. The SPHERE Wearable operates the accelerometer at 12 or $25 \mathrm{~Hz}$ and continuously communicates raw data over nonconnectable undirected BLE advertisements.

The SPHERE Wearable and Environmental Sensors communicate their data to a home server, named SPHERE Home Gateway (SHG), over a backbone 6LoWPAN network of mains-powered nodes, namely the SPHERE Forwarders. The SPHERE Forwarders are equipped with two radios. The primary radio is used to connect the device to the 6LoWPAN mesh network; the secondary operates as a BLE observer and collects the BLE advertisements that are broadcasted by the surrounding wearables. Effectively, the SPHERE Forwarders bridge the BLE and 6LoWPAN networks. Room-level indoor localization of the house residents is implemented by leveraging the reception of the BLE advertisement by multiple SPHERE Forwarders and fusing this information with the PIR data from the Environmental Sensors. The root SPHERE Forwarder, named the SPHERE IoT Gateway, is responsible for delivering all the data to the SHG.
Lastly, the SPHERE platform employs commercial off-theshelf appliance monitors to track the usage of appliances that are associated with important activities of daily life, such a TV, a microwave or a kettle. The appliance monitors communicate to a receiver node, attached to the SHG over a proprietary RF stack at $434 \mathrm{MHz}$.

\section{Data Aggregation and System Monitoring}

In deployment, the SPHERE system collects two broad categories of data: (i) sensor data from each modality and (ii) monitoring data. All data samples and accompanying metadata are stored securely at each participant's premises and will be collected at the end of each deployment. A sub-set of system monitoring data is also sent to the SPHERE Data Hub over a $3 \mathrm{G} / 4 \mathrm{G}$ link. Only monitoring data is transmitted, for two reasons: firstly, our research ethics policy mandates this step; secondly, sending all data collected by SPHERE to the Data Hub would require the transmission of multiple gigabytes per day over the $3 \mathrm{G} / 4 \mathrm{G}$ link, dramatically increasing cost.

The SPHERE Home Gateway collects the sensor data from home sensors (Fig. 2). Health-related raw sensor data and other personal data are stored in an encrypted, external, 1 TB solidstate drive. The SHG hosts an ActiveMQ MQTT (Message Queuing Telemetry Transport) broker which facilities MQTT communication. The SHG is also responsible for processing the incoming streams of data, making sure that the overlaying machine learning frameworks are provided with homogeneous data streams. Lastly, the SHG hosts a user interface website, namely the SPHERE Genie, that allows the participants to control the system (e.g., temporarily stop data collection, delete data, etc.), and is also capable of generating alerts and reminders to participants on behalf of SPHERE. Following a user-led design process, the participants opted for a tablet for accessing the SPHERE Genie.

Monitoring data includes a filtered subset of self-reported IoT sensor metrics received through MQTT alongside a parallel infrastructural monitoring solution based on Nagios. In the latter, the SHG actively polls various devices within the home network, pushing data regularly back to the Data Hub. The use of MQTT-based reporting infrastructure is consistent with best practice in IoT environments [12]. There are two primary motivations behind this monitoring combination. Firstly, a SPHERE installation combines IoT devices with relatively traditional home networking that also requires monitoring; secondly, each approach addresses a series of known risks. Analysed through a process structuring model for fault-tolerant application development, MQTT is operationally complex to receive, process, filter and forward, requiring several processes with non-trivial dependencies, any of which may develop a fault. Each process is supervised via a hardware watchdog or software supervisor, and automated recovery is triggered when a fault is identified. However, flaws may arise that are not detected by these supervisors (for example, a previously undetected or novel bug). The use of a maximally operationally independent monitoring system allows for a level of redundancy - a second opinion. This information is transmitted via Nagios Remote Data Processor, a straightforward XMLover-HTTPS-based data transport mechanism. 


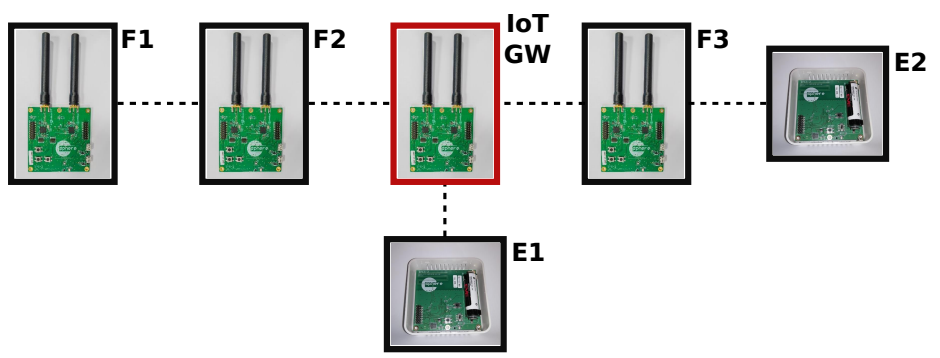

(a)

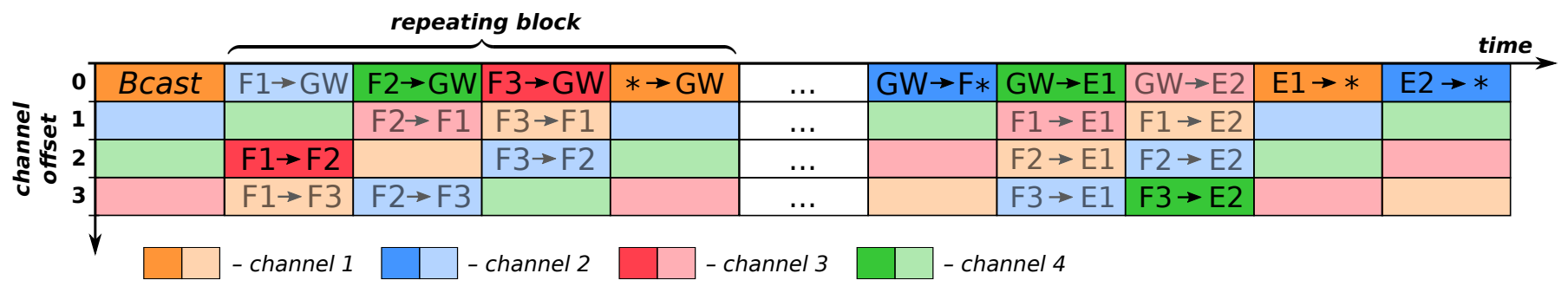

(b)

Figure 3: Scheduling in SPHERE: a) SPHERE IoT network with six nodes, b) SPHERE schedule for that network. BCast denotes a common timeslot reserved for broadcast, $* \rightarrow G W$ and $G W \rightarrow F *$ slots shared between multiple nodes.

Each home deployment accesses a private APN (Access point name) over $3 \mathrm{G} / 4 \mathrm{G}$, authenticating via a combination of APN credentials and SIM/device whitelisting. Using a private APN protects deployed devices from external attack, such as exploitation of router firmware vulnerabilities, and provides a mechanism for the implementation of security restrictions, such as whitelisting of destination IP addresses for outgoing messages. These measures also protect the project from theft and abuse of deployed SIMs, and adhere to the "principle of least privilege" : the home deployment receives only sufficient network access privilege to complete its intended functions. From this point, messages are routed over an encrypted channel to a load balancer fronting a segment of the University network. This routes each incoming connection to an operative data hub endpoint, permitting higher quality-of-service than a single endpoint allows and offering resilience to certain types of service outage.

Moving forward, 5G aims to increase the capacity of mobile communication systems and to enable massive machine communications. Future versions of the SPHERE system could leverage that capability in order to transmit most sensor and monitoring data at runtime, while potentially increasing data sampling rates (subject to battery lifetime restrictions). In a 5G context, Low-Power WAN technologies, such as for example NarrowBand IoT (NB-IoT), can be deployed as a back-channel to deliver the most essential monitoring data during outages of the primary $3 \mathrm{G} / 4 \mathrm{G}$ channel.

\section{IoT Networking Protocols}

SPHERE uses 6LoWPAN for the environmental sensor network and for data forwarding, and BLE to collect the data from the wearable nodes. This selection is made as 6LoWPAN has better support for multihop mesh, while BLE is more convenient for mobile nodes. 6LoWPAN allows to have globally unique, automatically generated IPv6 addresses as node identifiers, to compress IPv6 headers, and to use RPL (Routing over Low Power and Lossy Networks) for routing.

At the 6LoWPAN network layer, SPHERE uses IPv6 on top of IEEE 802.15.4 TSCH (i.e., 6TiSCH). We believe that the use of TSCH is instrumental to the early success of the SPHERE's deployments. TSCH provides time synchronization to the network and is essential in timestamping all of sensor data with high accuracy. A custom high-datarate TSCH schedule is used (Fig. 3b], tailored for SPHERE traffic patterns, as described in detail in [13]. The schedule, which defines all potentially active cells, is statically defined in the code of the nodes. At the start of each active TSCH slot, a node wakes up and dynamically selects which cell to use depending on its RPL routing state. Figure $3 \mathrm{~b}$ shows this through displaying the cells active in the network (Fig. 3a) in darker colors. In this way, topology changes are handled in a highly dynamic way, without needing to rebuild the schedule, unlike in other TSCH scheduling solutions. Furthermore, we use timeslot overallocation to accommodate traffic bursts due to retransmissions.

The schedule is restrictive in terms of maximal number of nodes. However, it is well-suited for the shallow networks typical in SPHERE where the average path length is less than two hops. The deployed SPHERE networks have one or two SPHERE IoT Gateways, eight to ten SPHERE Environmental Sensor nodes, and two to five SPHERE Forwarders that collect data from one to four SPHERE Wearables. Higher numbers of wearables are supported if either the number of gateways is increased, the number of forwarders decreased, or the sampling rate decreased.

The application layer is based on CoAP (Constrained Application Protocol), which supports both the client-server model through client-initiated queries for CoAP resources, and the subscribe-notify model through the CoAP observe 
functionally. The later refers to a communication pattern where a client requests to observe a resource and in turn the server notifies the client autonomously, either periodically or in an event-driven fashion. Environmental sensor data, along with acceleration data collected by the SPHERE Forwarders and system monitoring data, constitute observable CoAP resources with parameters that can be remotely controlled, such as the sampling period.

SPHERE opted to use CoAP instead of MQTT inside the sensor network as a more light-weight option. The SPHERE Home Gateway runs a daemon that functions as a translator between CoAP and MQTT.

The source code of SPHERE is built on Contiki, an opensource IoT OS that provides an open source implementation of TSCH and 6TiSCH, and Texas Instruments RTOS, an embedded OS that provides a free-to-use BLE stack.

\section{Challenges}

To support the requirements described below, SPHERE's approach is twofold: we leverage the above mentioned standardized protocols and state-of-the-art embedded operating systems, and we extend them with project-specific enhancements where the out-of-the-box solution is insufficient. This results in a unique system that advances the state-of-the-art in the following aspects:

\section{A. Dependability}

Attention to dependability is paid at multiple levels. For example, to reliably recover from software problems on sensor nodes, the SPHERE devices have two hardware watchdog timers. On the SHG, a supervisor agent periodically checks the gateway script's health status and restarts it in case it appears to be malfunctioning.

In terms of networking, the IEEE 802.15.4 TSCH protocol is critical for the reliability of the system, as it achieves high packet delivery rate due to its use of pseudorandom frequencyhopping and high predictability because of its scheduled nature. We additionally enhance the $\mathrm{TSCH}$ protocol with adaptive MAC-layer frequency channel selection. By continuously estimating per-channel background noise levels, the system is able to avoid the frequencies that are heavily interfered by WiFi or other sources. This increases the link-layer packet reception rate and reduces the number of retransmissions, further increasing the end-to-end packet delivery rate. On top of that, randomization in the initial channel hopping sequence is used to avoid extensive packet collisions in case of multiple overlapping SPHERE networks.

Baseline TSCH already compares favorably with other IoT protocols such as ZigBee, which was used in the first version of the SPHERE [9], since ZigBee only uses a single channel at a time and does not have timeslots. Our enhancements to TSCH also make it more robust than BLE advertisements, which use channel hopping, but not adaptive channel selection.

The results from the first 12 SPHERE deployments (Fig. 4) confirm high packet delivery rate in the TSCH network even in houses that are affected by high levels of WiFi interference in some channels (Fig. 5).

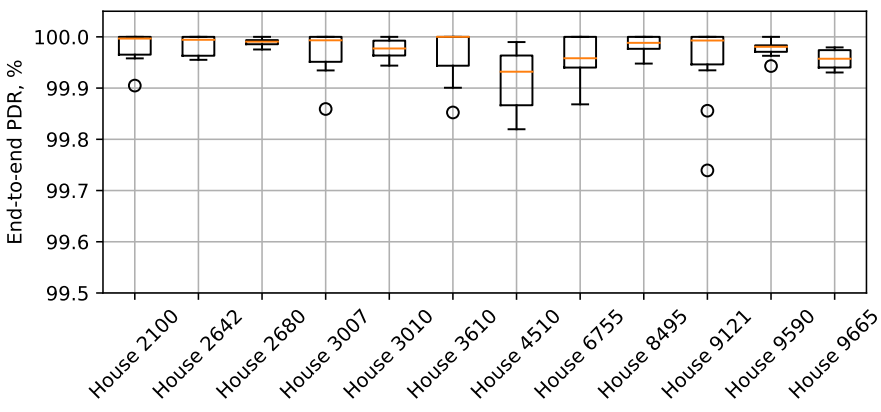

Figure 4: Reliability of the SPHERE networks in the 100homes deployment. Data from the first 12 public deployments, for July 21-28, 2017. All nodes show better than 99.7 percent delivery rate (average: 99.97 percent).

\section{B. High throughput}

The system has to forward and collect high-rate data from multiple accelerometers, therefore has much higher data-rate requirements compared to the classical low-power wireless systems for environmental monitoring. In particular, each SPHERE Forwarder must forward accelerometer data from up to four SPHERE Wearables. Furthermore, the throughput must account for multi-hop forwarding from other gateways and for packet retransmissions; in particular, SPHERE aims to build a reliable application layer network on top of a 50 percent or higher link-layer packet reception rate.

With these requirements, each forwarder node may use up to $20 \mathrm{TSCH}$ slots per second out of the 100 total. Assuming the TSCH slots on the IoT gateway node are fairly divided among all forwarders in the network, the system may support only up to four forwarder nodes. To circumvent this limitation, the SPHERE TSCH is enhanced with two features:

1) Shared slots in the schedule, which allow to have asymmetric packet rates from different forwarders without rebuilding the schedule at the run-time [13];

2) The option to have two SPHERE IoT Gateways in a single system for load-balancing reasons. If this feature is enabled, twice as many forwarders may be deployed. Furthermore, the dual gateway approach decreases the expected number of retransmissions, as in our experiments even co-located gateways with their antennas separated just by $5 \mathrm{~cm}$ show up to $8 \mathrm{~dB}$ difference in signal strength due to multipath induced fading. Therefore a single dual-radio SPHERE Forwarder node can function as two logical ones - the main and the backup gateway simultaneously.

These features enable a higher cumulative packet generation rate $(8$ forwarder nodes, each generating 14 packets per second show $>99.997$ percent PDR in a test house) than offered by commercial TSCH-based solutions [14].

\section{Energy efficiency}

Battery-replacement must be minimized, similar to other maintenance operations. SPHERE Wearables are wirelessly rechargeable; users are instructed to recharge them on a monthly basis, or alternatively when the SPHERE graphical 


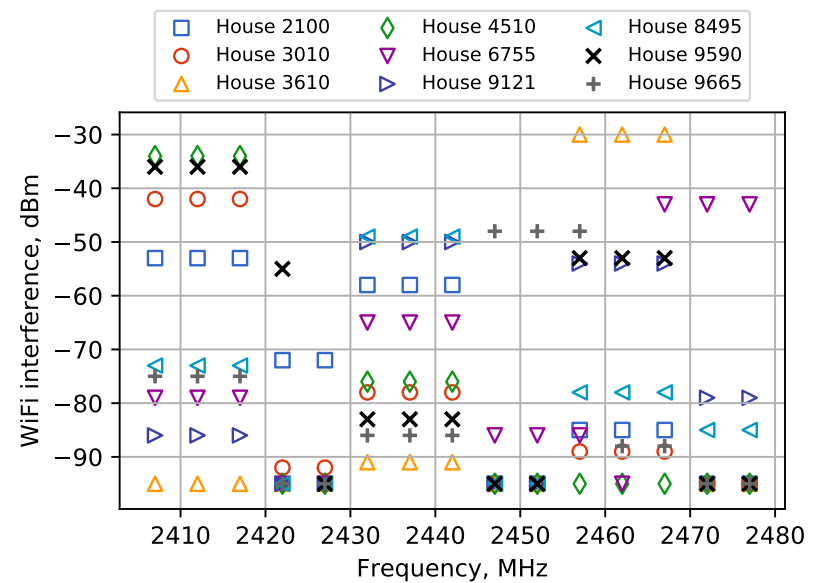

Figure 5: WiFi interference levels in the SPHERE houses. Data from nine houses taken on pre-deployment site-surveys.

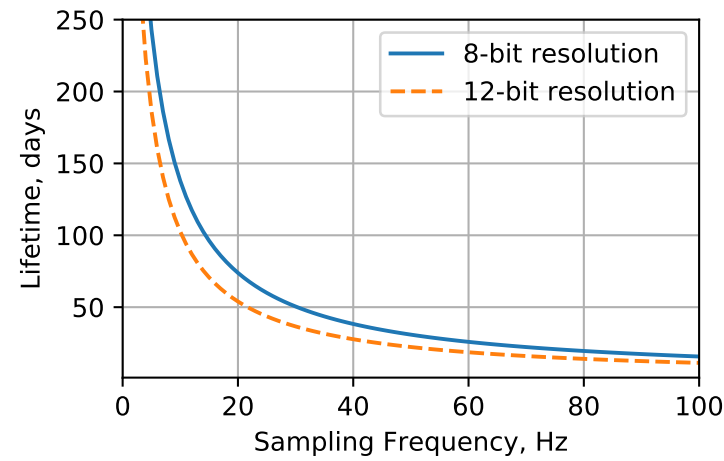

Figure 6: Battery lifetime of Wearable Sensors depending on load, given a $100 \mathrm{mAh}$ battery.

user interface alerts them. SPHERE Environmental Sensors are not rechargeable and ideally should function for the duration of the deployment (up to one year) without battery replacement.

The SPHERE sensing platforms are designed to operate efficiently when they are active and yield a very low idle power when inactive. As the result, SPHERE sensing platforms yield large battery lifetimes, as shown in Fig. 6 By employing state-of-the-art components (e.g., CC2650) and a minimalistic BLE advertisements based link-layer, the SPHERE Wearable Sensors can operate for several months on a single charge [15] when transmitting raw acceleration data at 12 or $25 \mathrm{~Hz}-$ an order-of-magnitude improvement compared with previous generation wearables such as SHIMMER, which lasts hours or days depending on its duty cycle [8].

The SPHERE Environmental Sensors use adaptive packet transmissions - a sensor measurement is transmitted to the gateway only when a significant change compared to the previous measurement is detected. Additionally, the sensors use an efficient binary data format as the CoAP payload; this allows to significantly increase the amount of data per packet compared to standard protocols like CBOR (Concise Binary Object Representation). These optimizations cumulatively lead to up to 7.5 times fewer packets, and allow to achieve long battery life.

\section{CONCLUSION}

This article highlights practical challenges, system requirements and lessons learned on designing and deploying in-thewild SPHERE's IoT network infrastructure for healthcare in home environment and reports initial results from the first 12 deployments. The initial results show good low-power wireless network performance ( 99.97 percent average PDR) despite interference from external WiFi networks. The SPHERE system is planned to be deployed to 100 properties and the data will be released after the end of the project.

\section{ACKNOWLEDGMENTS}

This work was performed under the SPHERE IRC funded by the UK Engineering and Physical Sciences Research Council (EPSRC), Grant EP/K031910/1.

\section{REFERENCES}

[1] E. S. Ford, J. B. Croft, S. F. Posner, R. A. Goodman, and W. H. Giles, "Co-Occurrence of Leading Lifestyle-Related Chronic Conditions Among Adults in the United States, 2002-2009," Prev Chronic Dis, vol. 10, pp. 1-12, Apr 2013.

[2] G. Livingston, B. Blizard, and A. Mann, "Does sleep disturbance predict depression in elderly people? A study in inner London." Br J Gen Pract, vol. 43, no. 376, pp. 445-448, 1993.

[3] R. Pfeffer, T. Kurosaki, C. Harrah Jr, J. Chance, and S. Filos, "Measurement of functional activities in older adults in the community," $J$. Gerontology, vol. 37, no. 3, pp. 323-329, 1982.

[4] C. E. Turcu and C. O. Turcu, "Internet of Things as Key Enabler for Sustainable Healthcare Delivery," Procedia - Social and Behavioral Sciences, vol. 73, pp. 251-256, 2013.

[5] P. Woznowski, A. Burrows, T. Diethe et al., "SPHERE: A Sensor Platform for Healthcare in a Residential Environment," in Designing, Developing, and Facilitating Smart Cities: Urban Design to IoT Solutions. Springer International Publishing, 2017, pp. 315-333.

[6] T. Watteyne, V. Handziski, X. Vilajosana, S. Duquennoy, O. Hahm, E. Baccelli, and A. Wolisz, "Industrial Wireless IP-Based Cyber-Physical Systems," Proc. IEEE, vol. 104, pp. 1025-1038, 2016.

[7] S. R. Islam, D. Kwak, M. H. Kabir, M. Hossain, and K.-S. Kwak, "The internet of things for health care: a comprehensive survey," IEEE Access, vol. 3, pp. 678-708, 2015.

[8] J. Ko, C. Lu, M. B. Srivastava, J. A. Stankovic, A. Terzis, and M. Welsh, "Wireless sensor networks for healthcare," Proc. IEEE, vol. 98, no. 11, pp. 1947-1960, 2010.

[9] N. Zhu, T. Diethe, M. Camplani, L. Tao, A. Burrows, N. Twomey, D. Kaleshi, M. Mirmehdi, P. Flach, and I. Craddock, "Bridging e-Health and the Internet of Things: The SPHERE project," IEEE Intell. Syst., vol. 30, no. 4, pp. 39-46, 2015.

[10] A. Elsts, G. Oikonomou, X. Fafoutis, and R. Piechocki, "Internet of Things for smart homes: Lessons learned from the SPHERE case study," in Proc. Global Internet of Things Summit (GIoTS), June 2017, pp. 1-6.

[11] X. Fafoutis, A. Elsts, R. Piechocki, and I. Craddock, "Experiences and Lessons Learned from Making IoT Sensing Platforms for Large-Scale Deployments," IEEE Access, vol. 6, pp. 3140-3148, 2018.

[12] A. Krylovskiy, M. Jahn, and E. Patti, "Designing a smart city internet of things platform with microservice architecture," in Proc. Int. Conf. Future Internet of Things and Cloud (FiCloud). IEEE, 2015, pp. 25-30.

[13] A. Elsts, X. Fafoutis, J. Pope, G. Oikonomou, R. Piechocki, and I. Craddock, "Scheduling High-Rate Unpredictable Traffic in IEEE 802.15. 4 TSCH Networks," in Proc. Int. Conf. Distributed Computing in Sensor Systems (DCOSS). IEEE, 2017, pp. 3-10.

[14] "SmartMesh Power and Performance Estimator," http://www.linear.com/ docs/42452. Linear Technology. Accessed on 30th April, 2018.

[15] X. Fafoutis, A. Vafeas, B. Janko, R. S. Sherratt, J. Pope, A. Elsts, E. Mellios, G. Hilton, G. Oikonomou, R. Piechocki, and I. Craddock, "Designing Wearable Sensing Platforms for Healthcare in a Residential Environment," EAI Endorsed Trans. Pervasive Health and Technology, vol. 17 , no. 12 , pp. 1-11, Sept. 2017. 
Atis Elsts (atis.elsts@bristol.ac.uk) [M'17] received his doctoral degree in computer science from the University of Latvia, in 2014. Since 2016 he is a researcher in the SPHERE interdisciplinary research collaboration at the University of Bristol. Prior to that, he was a researcher at Uppsala University and at RISE SICS. His main scientific interest is experimental research in Networked Embedded Systems. He is also a maintainer of the Contiki-NG operating system for the IoT.

Xenofon Fafoutis (xefa@dtu.dk) [S'09-M'14] received a PhD in Embedded Systems Engineering from the Technical University of Denmark (2014); an MSc in Computer Science from the University of Crete (2010); and a BSc in Informatics and Telecommunications from the University of Athens (2007). Since 2018, he is an Assistant Professor with the Technical University of Denmark. His research interests are in the fields of Networked Embedded Systems and the Internet of Things (IoT).

Przemyslaw Woznowski (p.r.woznowski@bristol.ac.uk) received his BSc and $\mathrm{PhD}$ degrees from Cardiff University in 2009 and 2013, respectively. He is currently a Senior Research Associate with the University of Bristol. His research interests are in the areas of IoT, wireless sensor network middleware (WSNM), eHealth, semantic web, ground-truth acquisition, logical modelling and reasoning. As a member of SPHERE, he is interested in serialising the vision of ubiquitous computing.

Emma Tonkin (e.1.tonkin@bristol.ac.uk@bristol.ac.uk) is a researcher at the University of Bristol. Originally educated as a physicist, she holds degrees in human-computer interaction and classical studies. In 2015, she was awarded a PhD from the University of Bristol on grounded context-awareness. Her interests include user-focused classification research, mining of data from the social web, secure large-scale data analysis and visualisation, and distributed network monitoring.

George Oikonomou (g.oikonomou@bristol.ac.uk) received the MSc Degree in Information Systems and the $\mathrm{PhD}$ degree in computer science from the Athens University of Economics and Business, Athens, Greece, in 2002 and 2009 respectively. He is currently a Lecturer with the Department of Electrical and Electronic Engineering at the University of Bristol. His current research focuses on energy-efficient networking for networks of severely constrained wireless embedded devices and the Internet of Things.

Robert Piechocki (r.j.piechocki@bristol.ac.uk) received an MSc degree from Technical University of Wroclaw (Poland) in 1997 and a PhD degree from the University of Bristol in 2002. He is currently a Reader in Advanced Wireless Access with the University of Bristol. His research interests span the areas of Statistical Signal Processing, Information and Communication Theory, Wireless Networking, Body and ad-hoc networks, Ultra Low Power Communications and Vehicular Communications.

Ian Craddock (ian.craddock@bristol.ac.uk) [M'09-SM'10-F'16] is currently a full Professor with the University of Bristol (UK) and Director of the flagship "SPHERE" centre (www.irc-sphere.ac.uk) comprising approximately 100 researchers and clinicians working on IoT technology for health. He serves on the healthcare strategy board for the UKs largest engineering funder. $\mathrm{He}$ is also separately employed by Toshiba as Managing Director of their Telecommunications Research Lab in Bristol. 